\title{
Laboratory diagnosis for malaria in the elimination phase in China: efforts and challenges
}

\author{
Jianhai Yin (凹), Mei Li, He Yan, Shuisen Zhou, Zhigui Xia (凶) \\ National Institute of Parasitic Diseases, Chinese Center for Disease Control and Prevention (Chinese Center for Tropical Diseases \\ Research); NHC Key Laboratory of Parasite and Vector Biology; WHO Collaborating Center for Tropical Diseases; National Center for \\ International Research on Tropical Diseases, Shanghai 200025, China
}

(C) Higher Education Press 2022

\begin{abstract}
Malaria remains a global health challenge, although an increasing number of countries will enter preelimination and elimination stages. The prompt and precise diagnosis of symptomatic and asymptomatic carriers of Plasmodium parasites is the key aspect of malaria elimination. Since the launch of the China Malaria Elimination Action Plan in 2010, China has formulated clear goals for malaria diagnosis and has established a network of malaria diagnostic laboratories within medical and health institutions at all levels. Various external quality assessments were implemented, and a national malaria diagnosis reference laboratory network was established to strengthen the quality assurance in malaria diagnosis. Notably, no indigenous malaria cases have been reported since 2017 , but the risk of re-establishment of malaria transmission cannot be ignored. This review summarizes the lessons about malaria diagnosis in the elimination phase, primarily including the establishments of laboratory networks and quality control in China, to better improve malaria diagnosis and maintain a malariafree status. A reference is also provided for countries experiencing malaria elimination.
\end{abstract}

Keywords malaria; laboratory diagnosis; quality control; malaria elimination; China

\section{Introduction}

Malaria is a life-threatening disease caused by parasites in the Plasmodium family that are transmitted to people through the bites of infected female Anopheles mosquitoes. Malaria is preventable and curable. At least five Plasmodium species are known to infect humans. Among them, $P$. falciparum and $P$. vivax are the most prevalent, and $P$. falciparum is the most virulent.

Despite global efforts to control and eliminate malaria, malaria remains a global health challenge. Nearly half of the world population is at risk of this disease according to the latest world malaria report, and the estimated number of malaria cases was 229 million in 2019 [1], compared with 228 million cases in 2018 [2]. In 2019, 409000 malaria deaths were recorded worldwide. Children under 5 years old accounted for $67 \%$ of all malaria deaths, and they have always been the most vulnerable group [1].

Received June 21, 2021; accepted August 10, 2021

Correspondence: Jianhai Yin, yinjh@nipd.chinacdc.cn; ZhiguiXia,xiazg@nipd.chinacdc.cn
In China, malaria records date back to approximately 4000 years ago. The four human Plasmodium species were distributed, primarily $P$. vivax and P. falciparum. Malaria spread countrywide, especially in rural areas, and outbreaks occurred frequently in the past $[3,4]$. Fortunately, the Chinese government initiated the national malaria elimination program in 2010 [5]. It aimed to achieve malaria elimination by 2020 , in line with the Millennium Development Goals. Since 2017, no indigenous malaria cases have been reported in China [6], and all 24 of the former endemic provinces passed the verification of subnational malaria elimination by June 2020. China has also been certified malaria free by the World Health Organization (WHO) on June 30, 2021 [7].

This achievement is inseparable from a strong malaria surveillance and response system, and the accurate and prompt diagnosis of malaria cases is the key aspect of the control and elimination of this disease. Even after its elimination, identifying the focus in a timely manner and carefully preventing malaria reintroduction will become more important [8]. This review focuses on the laboratory diagnosis of malaria in the elimination phase in China and systematically reviews the experience of China in 
developing a malaria diagnosis reference laboratory network, maintaining malaria microscopy competency, and implementing the quality control of malaria microscopy and nucleic acid testing, among others, to provide a reference for countries experiencing malaria elimination.

\section{Framework of malaria diagnostic laboratory network}

To reduce the overuse of antimalarial drugs and reduce the drug pressure on parasites, all suspected malaria patients should be confirmed by parasite-detection methods such as quality-assured microscopy or a rapid diagnostic test before receiving antimalarial treatment, according to the WHO recommendations [9].

Strengthening the diagnosis and treatment in all settings will help reduce the morbidity and mortality of malaria. Expanding access to timely diagnostic testing will provide prompt and accurate surveillance data based on confirmed rather than suspected cases. These actions will lead to an improvement in the identification and management of the many nonmalarial febrile illnesses presumed to be malaria based on the presence of fever alone.
In China, public health services are primarily provided by professional public health service institutions, primary medical and health institutions, and general hospitals at different levels. A malaria diagnostic laboratory network based on these institutions helps to eliminate malaria. The centers for disease control and prevention (CDCs) are responsible for the implementation, technical guidance, and professional training in malaria elimination. More importantly, the CDCs perform malaria surveillance and response, including case laboratory reviews, case epidemiological investigations and classifications, foci investigations and disposal, and technical training. Additionally, various medical institutions at all levels are responsible for case identification, reporting, and treatment. Health facilities at the county and prefectural levels are the most concentrated facilities for the initial diagnosis and confirmation of malaria [10].

Accordingly, China has set clear targets for blood tests for the detection of malaria parasites at all levels and types of medical and health institutions and at entry-exit inspection and quarantine agencies (Table 1) to achieve the goal of malaria elimination by 2020 as described in China Malaria Elimination Action Plan (2010-2020) [5]. The capacity and responsibility requirements in malaria

Table 1 Objectives of blood tests for Plasmodium in fever patients in China Malaria Elimination Action Plan (2010-2020)

\begin{tabular}{|c|c|c|c|c|}
\hline Year & $\begin{array}{l}\text { A proportion of institutions can } \\
\text { perform blood tests for malaria } \\
\text { parasite }\end{array}$ & $\begin{array}{l}\text { Annual blood examination } \\
\text { for malaria parasites in patients } \\
\text { with clinically diagnosed } \\
\text { malaria, suspected malaria, } \\
\text { and unexplained fever }\end{array}$ & Laboratory test & $\begin{array}{l}\text { Malaria screening by entry- } \\
\text { exit inspection and quarantine } \\
\text { agencies }\end{array}$ \\
\hline By 2012 & $\begin{array}{l}\text { General hospitals and CDCs at } \\
\text { the provincial, prefecture level } \\
\text { reach } 100 \% \\
\text { General hospitals and CDCs in } \\
\text { the Category I, II, and III } \\
\text { counties reach } 100 \% \\
\text { Township medical institutions } \\
\text { in Category I and II counties } \\
\text { reach } 90 \%\end{array}$ & $\begin{array}{l}\text { Not be less than } 2 \% \text { and } 1 \% \\
\text { of the population in the } \\
\text { jurisdiction of towns and } \\
\text { townships in Category I and } \\
\text { II counties, respectively } \\
\text { Not be less than } 2 \% \text { of the } \\
\text { population in the jurisdiction } \\
\text { of Category III counties } \\
\text { Tests in the malaria } \\
\text { transmission season should } \\
\text { not be less than } 80 \%\end{array}$ & $\begin{array}{l}\text { Laboratory test rate of malaria } \\
\text { cases reaches } 100 \% \\
\text { Laboratory confirmed rate } \\
\text { reaches } 75 \%\end{array}$ & $\begin{array}{l}100 \% \text { of fever patients entering } \\
\text { from overseas malaria endemic } \\
\text { areas }\end{array}$ \\
\hline By 2015 & $\begin{array}{l}\text { General hospitals and CDCs at } \\
\text { the provincial, prefecture level } \\
\text { remain } 100 \% \\
\text { General hospitals and CDCs in } \\
\text { the Category I, II, and III } \\
\text { counties remain } 100 \% \\
\text { Township medical institutions } \\
\text { in Category I and II counties } \\
\text { reach } 100 \%\end{array}$ & $\begin{array}{l}\text { Not be less than } 1 \% \text { and } 2 \% \text { of } \\
\text { the population in the jurisdiction } \\
\text { of towns and townships in } \\
\text { Category I and II counties, } \\
\text { respectively } \\
\text { Not be less than } 2 \% \text { of the } \\
\text { population in the jurisdiction } \\
\text { of Category III counties } \\
\text { Tests in the malaria } \\
\text { transmission season should } \\
\text { not be less than } 80 \%\end{array}$ & $\begin{array}{l}\text { Laboratory confirmed rate } \\
\text { reaches } 100 \%\end{array}$ & $\begin{array}{l}100 \% \text { of fever patients entering } \\
\text { from overseas malaria endemic } \\
\text { areas }\end{array}$ \\
\hline Ву 2020 & $\begin{array}{l}\text { Medical and health institutions } \\
\text { and CDCs at the county level } \\
\text { and above in the original } \\
\text { endemic counties all have } \\
\text { facilities and capabilities } \\
\text { for blood testing of malaria } \\
\text { parasites }\end{array}$ & & $\begin{array}{l}\text { All people suspected of malaria } \\
\text { get a laboratory blood test for } \\
\text { malaria parasites; malaria cases } \\
\text { whose source of infection cannot } \\
\text { be determined by epidemiology } \\
\text { should be genetically traced and } \\
\text { identified by national laboratories }\end{array}$ & \\
\hline
\end{tabular}


diagnosis in laboratories at different levels were also described. Specifically, the competency assessment and quality assurance of malaria diagnosis performed by technicians in clinical laboratories and laboratory staff for malaria diagnosis in CDCs at different levels were emphasized.

\section{Progress of the national malaria diagnosis reference laboratory network}

In the beginning of the national malaria elimination program, the results of malaria diagnosis in various laboratories from different provinces under different conditions were not related, and no basis for comparison was apparent. Therefore, a center or reference laboratory was urgently needed. Work on this laboratory should be conducted under fair conditions, and it should serve the public, where fixed standards can be maintained. As a result, the China malaria diagnosis reference laboratory network with a quality management system based on provincial CDCs/IPDs (institute of parasitic diseases) has been set up since 2011 [11]. It covers 25 provinces, including all 24 historical malaria-endemic provinces and one nonendemic province $[12,13]$.

A provincial reference laboratory that wants to join the network needs to pass the review of an independent expert group. The review includes the hardware conditions of the laboratory (including the laboratory's spatial layout, facilities, etc.) and software conditions (including personnel composition, funding guarantees, personnel competency, etc.). Furthermore, the qualified competency to detect malaria parasites, including microscopy and the nucleic acid amplification test (NAAT), plays a key role in the review, and the laboratory cannot be included into the network if it cannot pass the competency assessment [11]. Furthermore, this network was established in stages: the provincial laboratories in Hainan, Yunnan, Henan, Jiangsu, and Shanghai were established in 2011; those in Zhejiang, Anhui, Shandong, Guangdong, Guangxi, Guizhou, Chongqing, and Gansu were approved in 2012; those in Liaoning, Shaanxi, Hubei, Hunan, Sichuan, and Fujian were approved in 2013; those in Shanxi, Hebei, and Xinjiang were approved in 2014; that in Jiangxi was approved in 2015; that in Tibet as the last one in the malaria-endemic areas was approved in 2016; and that in Ningxia as the first one in nonmalaria endemic areas was approved in Feb 2021.

In this network, the national and provincial reference laboratories are primarily responsible for case review, malaria diagnosis capacity training, and quality assurance of the performance of diagnostic assays used in clinics and in the field to ensure the accuracy and reliability of the results [11]. The network has indeed played an important role in laboratory diagnosis. Since its establishment, the country's clinically diagnosed cases and unclassified malaria cases have dropped sharply [14-17] (Fig. 1), and no unclassified cases have been reported since 2013 [17].

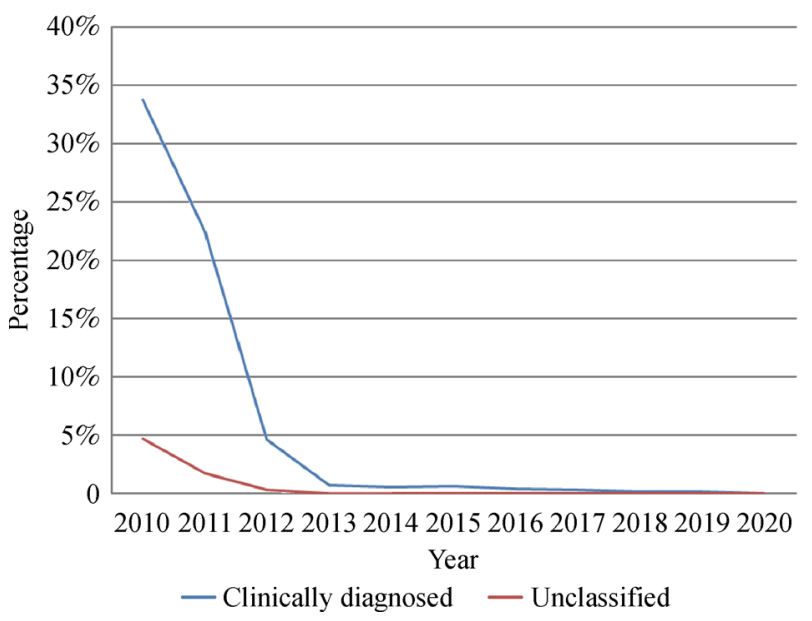

Fig. 1 Trends of proportion of clinically diagnosed and unclassified cases in the total malaria cases reported in China, 2010-2020.

\section{Competency in malaria parasite detection and quality control}

With the dramatic decline in indigenous malaria cases, competency in malaria diagnosis especially through microscopy is difficult to maintain. It is time consuming and experience dependent with low sensitivity, but it is still the gold standard method of diagnosing malaria [18]. For example, nearly $11 \%$ of the reported cases occurred in 2017-2018, and the final review of the results were inconsistent with the initially confirmed results [10]. Therefore, different types of quality assurances and control activities are conducted to improve competency in malaria diagnosis, which primarily comprises microscopy and polymerase chain reaction (PCR). The three diagnostic criteria in malaria, involving various cases and the processes for the preparation of blood smears including fixation, hemolysis, Giemsa staining, microscopy, and blood smear storage, were updated [19-21] (Table 2). Plasmodium NAAT was written in the Diagnosis of Malaria (WS259-2015) [20].

First, the annual national technique competition on the prevention and control of parasitic diseases has been held since 2011 to continuously consolidate and improve capacity building in CDCs/IPDs and medical institutions across the country [22,23]. Malaria-related theoretical knowledge, thick and thin blood film preparation, and identification and quantification of Plasmodium species using light microscopy are important components of the 
Table 2 Definition of malaria cases and infections in the diagnostic criteria

\begin{tabular}{|c|c|c|}
\hline Definition & Diagnostic Criteria for Malaria (WS259-2006) & Diagnosis of Malaria (WS259-2015) \\
\hline Carrier & $\begin{array}{l}\text { Asymptomatic } \\
\text { Test positive: microscopy }\end{array}$ & - \\
\hline Suspected case & $\begin{array}{l}\text { Epidemiological history of malaria } \\
\text { Atypical symptoms of malaria }\end{array}$ & - \\
\hline Clinically diagnosed case & $\begin{array}{l}\text { Epidemiological history of malaria } \\
\text { Typical symptoms of malaria, or atypical symptoms of } \\
\text { malaria; and presumptive treatment is effective }\end{array}$ & $\begin{array}{l}\text { History } \\
\text { Typical symptoms of malaria, or atypical symptoms of } \\
\text { malaria }\end{array}$ \\
\hline Confirmed case & $\begin{array}{l}\text { Epidemiological history of malaria } \\
\text { Clinical manifestations } \\
\text { Test positive: microscopy and/or RDTs }\end{array}$ & $\begin{array}{l}\text { Clinically diagnosed case } \\
\text { Test positive: microscopy and/or RDTs and/or NAATs }\end{array}$ \\
\hline Asymptomatic infection & - & $\begin{array}{l}\text { Asymptomatic } \\
\text { Test positive: microscopy and/or RDTs and/or NAATs }\end{array}$ \\
\hline Severe case & - & $\begin{array}{l}\text { Confirmed case } \\
\text { Severe symptoms }\end{array}$ \\
\hline
\end{tabular}

a Nucleic acid amplification test (NAAT) products for clinical diagnosis should be registered and approved by the State Food and Drug Administration. The laboratory operation is conducted in strict accordance with the quality-control requirements of the national reference laboratory. If necessary, samples are stored for inspection by superior laboratory experts.

competition. This method is conducive to promoting training using a competition. Furthermore, the requirements for participants are strict, i.e., age not exceeding 45 years old, at least one participant from a medical institution, and at least two participants from CDCs at the county level. All qualified technicians could participate in the national competition only once [12]. However, the performance of Plasmodium species identification and parasite counting was far less than that of blood-smear preparation in previous competitions from 2011 to 2015 [12]. Many provinces also organize intensive training courses and provincial competitions according to similar requirements before the national competition.

Second, an effective model for evaluating the competency of microscopists in malaria, named the External Competency Assessment of Malaria Microscopists (ECAMM) courses, which were developed by the WHO [12,24-26], has been held no less than seven times in China since 2010. Microscopists from national and provincial laboratories were evaluated using an examination involving Plasmodium species identification and parasite counting. By the end of 2019, a total of 19 microscopists obtained a level 1 certificate, 15 obtained a level 2 certificate, 7 obtained a level 3 certificate, and 6 obtained a level 4 certificate. Each province has at least one malaria microscopist who has obtained a certificate. Such high-level microscopists are critical to the quality of malaria diagnosis in the elimination of malaria in various provinces, thereby positively contributing to the decreased numbers of indigenous malaria cases in certain areas in China [24].

Third, two other WHO external quality assessment (EQA) programs aimed at the competency of malaria microscopy and NAAT of the national laboratory were held in China [12,27]. In terms of malaria microscopy, the competency in Plasmodium species identification and parasite counting, similar to ECAMM, was assessed using 15 slides (20 slides used in Round 1, 2012) in each round of the EQA. As a result, positive and negative identification of slides and identification of $P$. falciparum were mostly accurate, but other species were easy to be confused $[12,27]$. However, the score of parasite quantification was unstable, and except for the scoring rate in the second round of 2013, which was lower than $60 \%$, the scoring rate in the other rounds was higher than $60 \%$, according to the commentaries for each round of EQA from the WHOWPRO/RITM EQA Programme for Communicable Diseases (Malaria Microscopy). Among them, the first round in 2014, the second round in 2017 and the first round in 2020 received full marks. For NAAT, the national laboratory participates in the global NAAT EQA scheme developed by the WHO Global Malaria Programme and the UK National External Quality Assessment Scheme (UK NEQAS) Parasitology. Ten specimens containing five major human-infecting Plasmodium species at various parasite densities and negative samples in the formats of lyophilized blood and dried blood spot were distributed in one round. Two distributions per year were sent [28]. The cumulative score of 36 out of a possible total of 40 , which was higher than the average of 29.58 in all laboratories in the past two rounds according to the results from UK NEQAS Parasitology for WHO Malaria Molecular Test in 2021 , indicated good competency of malaria NAAT in the national laboratory.

In general, blood samples tested for malaria parasites should be reviewed level by level. All positive samples must be verified at county, prefectural, and provincial laboratories, and no less than $10 \%$ of negative slides prepared in the clinics must be reviewed by the county CDCs. No less than $3 \%$ of the former and no less than $1 \%$ 
of the former again must be reviewed by the prefectural and provincial levels, respectively. Moreover, each positive sample should be verified by microscopy and PCR in provincial laboratories [11,29-35]. It provides important information for provinces in analyzing the malaria parasite-detection competencies of laboratories at all levels in a timely manner to ensure the accuracy of the results. Additionally, the national laboratory performs a NAAT verification or invites malaria microscopists with a WHO level 1 or 2 across the country to randomly check the above samples [36].

\section{Challenges and perspectives}

Although China has not reported indigenous cases of malaria since 2017, thousands of imported cases primarily suffer from $P$. falciparum and $P$. vivax every year $[6,37,38]$. Even in 2020 during the COVID-19 pandemic, 1085 imported cases were still recorded [39]. If these cases were not detected promptly, retransmission could potentially develop due to the wide distribution of suitable malaria vectors in the transmission season with the importation of malaria [37], particularly imported vivax malaria cases from South-east Asia.

The diagnostic tools usually used for malaria diagnosis clinically include light microscopy and rapid diagnostic tests (RDTs). Although they contribute to malaria control and elimination, these two methods have poor sensitivities in asymptomatic patients, resulting in the potential underestimation of malaria prevalence compared with molecular diagnostic tools such as PCR [40]. More importantly, persistent, asymptomatic Plasmodium infections carry health risks for infected individuals and provide a parasite reservoir for malaria transmission at the public health level $[41,42]$.

Furthermore, malaria RDTs based on $P$. falciparum histidine-rich protein 2 (HRP2) have encountered challenges in recent years. The HRP2/3 deletion strains of $P$. falciparum have been reported in malaria endemic areas since their first discovery in Peru in 2010, leading to a false negative RDT test that increases the risk of $P$. falciparum transmission [1,43-45]. Therefore, high-sensitivity tests such as the NAAT, which is recommended by the national malaria diagnosis reference laboratory network, should not be limited to sample review in provincial and national laboratories. Instead, it should be expanded to more medical and health institutions to detect low-density parasitemia and identify all infected individuals. Notably, the Chinese government clearly requires that tertiary general hospitals, infectious disease hospitals, CDCs at all levels, and at least one county-level hospital in the county must have the competency of nucleic acid testing by the end of September 2020. All secondary general hospitals must also have this competency by the end of
2020 to respond effectively to the COVID-19 pandemic [46]. It also provides for NAAT laboratories and a workforce for malaria diagnosis.

In the future, EQA activities regarding malaria diagnosis should be extended from microscopy to the NAAT. These activities should be regularly held to verify the quality of the NAAT in clinical, reference, and research laboratories and monitor their performances over time. Preparing and confirming EQA reference samples from in vitro cultures of laboratory strains or from leftover clinical specimens is important [28]. Alternatively, multiple samples can be collected and tested from a participating laboratory, and the results can be compared with those from participating laboratories.

Moreover, malaria deaths caused by imported $P$. falciparum have become a great challenge in China. Diagnosis delay is the main cause of malaria deaths due to the vagueness of malaria symptoms in patients and treatments, as well as the clinicians' insufficient experience in the diagnosis and treatment of malaria $[47,48]$. Very few malaria cases have been diagnosed and reported by customs $[10,49]$, although travel-related malaria infections are a key issue given the increase in international travel worldwide. Therefore, the malaria parasite screening of travelers upon arrival must be strengthened to promptly identify malaria infections (such as reactive and proactive case detection) in the post-elimination phase [50]. Furthermore, improvements in the sharing and exchange of information among customs, hospitals, and CDCs will improve the malaria vigilance of health workers. Evidencebased malaria-related health education for travelers will also contribute to timely malaria diagnoses and help maintain the malaria-free status of China.

\section{Acknowledgements}

This work was supported by the National Science and Technology Major Program of China (No. 2018ZX10101002-002), the Fifth Round of Three-Year Public Health Action Plan of Shanghai (No. GWV-10.1-XK13), and the Bill \& Melinda Gates Foundation (No. INV-018913).

\section{Compliance with ethics guidelines}

Jianhai Yin, Mei Li, He Yan, Shuisen Zhou, and Zhigui Xia declare that they have no financial conflicts of interest. This manuscript is a review article and does not involve a research protocol requiring approval by a relevant institutional review board or ethics committee.

\section{References}

1. WHO. World malaria report 2020: 20 years of global progress and challenges. Geneva: World Health Organization, 2020 
2. WHO. World malaria report 2019. Geneva: World Health Organization, 2019

3. Tang LH, Xu LQ, Chen YD. Parasitic Disease Control and Research in China. Beijing: Beijing Science \& Technology Press, 2012 (in Chinese)

4. Yin JH, Zhou SS, Xia ZG, Wang RB, Qian YJ, Yang WZ, Zhou XN. Historical patterns of malaria transmission in China. Adv Parasitol 2014; 86: 1-19

5. Ministry of Health, the People's Republic of China. China Malaria Elimination Action Plan (2010-2020). 2010 (in Chinese)

6. Zhang L, Feng J, Zhang SS, Xia ZG, Zhou SS. The progress of national malaria elimination and epidemiological characteristics of malaria in China in 2017. Chin J Parasitol Parasit Dis (Zhongguo Ji Sheng Chong Xue Yu Ji Sheng Chong Bing Za Zhi) 2018; 36(3): 201-209 (in Chinese)

7. WHO. From 30 million cases to zero: China is certified malaria-free by WHO. https://www.who.int/news/item/30-06-2021-from-30million-cases-to-zero-china-is-certified-malaria-free-by-who (accessed June 30, 2021)

8. malERA Consultative Group on Diagnoses and Diagnostics. A research agenda for malaria eradication: diagnoses and diagnostics. PLoS Med 2011; 8(1): e1000396

9. WHO. Universal access to malaria diagnostic testing: an operational manual. Geneva: World Health Organization, 2011

10. Yin J, Zhang L, Feng J, Zhou S, Xia Z. Malaria diagnosis and verification-China, 2017-2018. China CDC Wkly 2020; 2(17): 285-288

11. Yin JH, Yan H, Huang F, Li M, Xiao HH, Zhou SS, Xia ZG. Establishing a China malaria diagnosis reference laboratory network for malaria elimination. Malar J 2015; 14(1): 40

12. Yin J, Yan H, Li M, Ruan Y, Zhang X, Wang L, Cao C, Xia Z, Zhou S. Competency and challenges in malaria microscopy in China. Biosci Trends 2017; 11(6): 702-705

13. National Institute of Parasitic Diseases, China CDC. The pre-review of the provincial reference laboratory for malaria diagnosis in Ningxia. http://www.ipd.org.cn/view5005.html (in Chinese) (accessed November 3, 2020)

14. Zhou SS, Wang Y, Li Y. Malaria situation in the People's Republic of China in 2010. Chin J Parasitol Parasit Dis (Zhongguo Ji Sheng Chong Xue Yu Ji Sheng Chong Bing Za Zhi) 2011; 29(6): 401-403 (in Chinese)

15. Xia ZG, Yang MN, Zhou SS. Malaria situation in the People's Republic of China in 2011. Chin J Parasitol Parasit Dis (Zhongguo Ji Sheng Chong Xue Yu Ji Sheng Chong Bing Za Zhi) 2012; 30(6): 419-422 (in Chinese)

16. Xia ZG, Feng J, Zhou SS. Malaria situation in the People's Republic of China in 2012. Chin J Parasitol Parasit Dis (Zhongguo Ji Sheng Chong Xue Yu Ji Sheng Chong Bing Za Zhi) 2013; 31(6): 413-418 (in Chinese)

17. Zhang L, Feng J, Xia ZG. Malaria situation in the People's Republic of China in 2013. Chin J Parasitol Parasit Dis (Zhongguo Ji Sheng Chong Xue Yu Ji Sheng Chong Bing Za Zhi) 2014; 32(6): 407-413 (in Chinese)

18. Ding G, Zhu G, Cao C, Miao P, Cao Y, Wang W, Gu Y, Xu S, Wang $\mathrm{S}$, Zhou H, Cao J. The challenge of maintaining microscopist capacity at basic levels for malaria elimination in Jiangsu Province, China. BMC Public Health 2018; 18(1): 489
19. Ministry of Health, the People's Republic of China. Diagnostic Criteria for Malaria (WS 259-2006). 2006 (in Chinese)

20. National Health and Planning of the People's Republic of China. Diagnosis of malaria (WS 259-2015). 2015 (in Chinese)

21. National Health and Planning of the People's Republic of China. Microscopic examination of blood films for malaria parasites (WS/T 569-2017). 2017 (in Chinese)

22. Fu Q, Li SZ, Wang Q, Zhang L, Liu W, Zheng X, Zhang SS, Xia ZG, Zhou SS, Chen Z, Wang LY, Zhou XN. Report of analysis of National Technique Competition for Diagnosis of Parasitic Diseases in 2011-II Analysis of capabilities of Plasmodium detection. Chin J Parasitol Parasit Dis (Zhongguo Ji Sheng Chong Xue Yu Ji Sheng Chong Bing Za Zhi) 2012; 24(3): 274-278 (in Chinese)

23. Ruan Y, Tian T, Zhu Z, Hao Y, Zhang L, Zhu T, Wang L, Wang Q, Cao C, Li S, Zhou X. Assessing competence for helminthiases: a lesson learnt from national contest of parasitic diseases in China in 2012-2016. Acta Trop 2019; 198: 105078

24. Li M, Zhou H, Yan H, Yin J, Feng X, Xia Z, Zhou S. Analysis on external competency assessment for malaria microscopists in China. Malar J 2019; 18(1): 366

25. WHO. Malaria microscopy quality assurance manual (Version 1). Geneva: World Health Organization, 2009

26. WHO. Malaria microscopy quality assurance manual (Version 2). Geneva: World Health Organization, 2016

27. Yin J, Yan H, Li M, Fang W, Huang F, Jiang W, Zhou S, Xia Z. Malaria diagnosis reference laboratory: analysis of malaria detecting ability under microscope in the External Quality Assurance Program. Int J Med Parasit Dis (Guo Ji Yi Xue Ji Sheng Chong Bing Za Zhi) 2015; 42(3): 173-175 (in Chinese)

28. Cunningham JA, Thomson RM, Murphy SC, de la Paz Ade M, Ding XC, Incardona S, Legrand E, Lucchi NW, Menard D, Nsobya SL, Saez AC, Chiodini PL, Shrivastava J. WHO malaria nucleic acid amplification test external quality assessment scheme: results of distribution programmes one to three. Malar J 2020; 19(1): 129

29. Fei L, Shuang Z, Yi Y, Shan-Shan L, Yan T, Jing-Ru X, Yang Z. Assessment of malaria microscopy competency at primary health institutions in the Chongqing Municipality. Front Med (Lausanne) 2021; 8: 602442

30. Dong Y, Mao XH, Chen MN, Deng Y, Wang J, Xu YC. Quality analysis of laboratory malaria diagnosis in Yunnan Province during 2012-2014. Chin J Parasitol Parasit Dis (Zhongguo Ji Sheng Chong Xue Yu Ji Sheng Chong Bing Za Zhi) 2015; 33(3): 191-195 (in Chinese)

31. She DY, Wu JH, Lu LD, Huang YT, Geng Y, Zhang NH. Evaluation of the testing ability of city/prefecture-level malaria laboratories in Guizhou Province during 2014-2016. Chin J Parasitol Parasit Dis (Zhongguo Ji Sheng Chong Xue Yu Ji Sheng Chong Bing Za Zhi) 2017; 35(5): 466-471 (in Chinese)

32. Sun LC, Wu DN, Dong XR, Tu Z, Lin W, Xia J. Results of Hubei provincial malaria diagnostic reference laboratory from 2014 to 2018. Chin J Dis Control Prev (Zhonghua Ji Bing Kong Zhi Za Zhi) 2021; 25(1): 108-112 (in Chinese)

33. Deng Y, Dong Y, Xu YC, Mao XH, Chen MN. Quality and impact factors of malaria diagnosis in Yunnan provincial laboratory from 2015 to 2016. Chin J Zoonoses (Zhongguo Ren Shou Gong Huan Bing Xue Bao) 2018; 34(4): 337-342 (in Chinese)

34. Sun LC, Dong XR, Tu Z, Wu DN, Lin W, Zhou XR, Zhu H. Re- 
examination of malaria diagnosis in Hubei provincial malaria diagnostic reference laboratory from 2017 to 2019. Chin J Schisto Control (Zhongguo Xue Xi Chong Bing Fang Zhi Za Zhi) 2020; 32 (6): 631-634 (in Chinese)

35. Xu S, Gu YP, Cao YY, Wang WM, Tang JX, Li JL, Zhang MH, Zhou HY, Zhu GD, Cao J. Results analysis of Jiangsu provincial malaria diagnostic reference laboratory in 2017. Chin J Schisto Control (Zhongguo Xue Xi Chong Bing Fang Zhi Za Zhi) 2018; 30 (6): 630-634 (in Chinese)

36. National Institute of Parasitic Diseases, China CDC. The national quality assessment of malaria blood smear preparation and microscopic examination, 2020. http://www.ipd.org.cn/view5034. html (in Chinese) (accessed December 25, 2020)

37. Zhang L, Feng J, Zhang SS, Xia ZG, Zhou SS. Epidemiological characteristics of malaria and the progress towards its elimination in China in 2018. Chin J Parasitol Parasit Dis (Zhongguo Ji Sheng Chong Xue Yu Ji Sheng Chong Bing Za Zhi) 2019; 37(3): 241-247 (in Chinese)

38. Zhang L, Feng J, Xia ZG, Zhou SS. Epidemiological characteristics of malaria and progress on its elimination in China in 2019. Chin J Parasitol Parasit Dis (Zhongguo Ji Sheng Chong Xue Yu Ji Sheng Chong Bing Za Zhi) 2020; 38(2): 133-138 (in Chinese)

39. Zhang L, Feng J, Tu H, Yin J, Xia ZG. Malaria epidemiology in China in 2020. Chin J Parasitol Parasit Dis (Zhongguo Ji Sheng Chong Xue Yu Ji Sheng Chong Bing Za Zhi) 2021; 39(2): 195-199 (in Chinese)

40. Ayong L, Moukoko CEE, Mbacham WF. Diagnosing malaria: methods, tools, and field applicability. Methods Mol Biol 2019; 2013: 73-82

41. Bousema T, Okell L, Felger I, Drakeley C. Asymptomatic malaria infections: detectability, transmissibility and public health relevance. Nat Rev Microbiol 2014; 12(12): 833-840

42. Cheaveau J, Mogollon DC, Mohon MAN, Golassa L, Yewhalaw D, Pillai DR. Asymptomatic malaria in the clinical and public health context. Expert Rev Anti Infect Ther 2019; 17(12): 997-1010

43. Gamboa D, Ho MF, Bendezu J, Torres K, Chiodini PL, Barnwell JW, Incardona S, Perkins M, Bell D, McCarthy J, Cheng Q. A large proportion of $P$. falciparum isolates in the Amazon region of Peru lack pfhrp2 and pfhrp3: implications for malaria rapid diagnostic tests. PLoS One 2010; 5(1): e8091

44. WHO. False-negative RDT results and implications of new reports of $P$. falciparum histidine-rich protein $2 / 3$ gene deletions. Geneva: World Health Organization, 2017

45. Thomson R, Parr JB, Cheng Q, Chenet S, Perkins M, Cunningham J. Prevalence of Plasmodium falciparum lacking histidine-rich proteins 2 and 3: a systematic review. Bull World Health Organ 2020; 98(8): 558-568F

46. The State Council of the People's Republic of China. Notice on the Work Plan for Further Promoting the Capacity Building of the COVID-19 Nucleic Acid Amplification Testing Issued by the State Council's Joint Prevention and Control Mechanism in Response to the COVID-19. http://www.gov.cn/zhengce/content/2020-08/31/ content 5538788.htm (in Chinese) (accessed August 31, 2020)

47. Zhang L, Tu H, Zhou S, Xia Z, Feng J. Malaria deaths - China, 2011-2020. China CDC Wkly 2021; 3(17): 360-365

48. Li W, Zhang T, Xu X, Jiang J, Yu C, Tian C, Wang S, Lyu X, Liu Z. Problems associated with the diagnosis of imported malaria in Anhui Province, China. Am J Trop Med Hyg 2020; 102(1): 142146

49. Fang LQ, Sun Y, Zhao GP, Liu LJ, Jiang ZJ, Fan ZW, Wang JX, Ji Y, Ma MJ, Teng J, Zhu Y, Yu P, Li K, Tian YJ, Cao WC. Travelrelated infections in mainland China, 2014-16: an active surveillance study. Lancet Public Health 2018; 3(8): e385-e394

50. Gunasekera WMKTAW, Premaratne R, Fernando D, Munaz M, Piyasena MGY, Perera D, Wickremasinghe R, Ranaweera KDNP, Mendis K. A comparative analysis of the outcome of malaria case surveillance strategies in Sri Lanka in the prevention of reestablishment phase. Malar J 2021; 20(1): 80 